\title{
Numerical Estimation of Notch Stress Intensity Factors of Sharp V-Notches
}

\author{
Mirzaul Karim Hussain ${ }^{1, *}$, and K.S.R.K. Murthy ${ }^{2}$ \\ ${ }^{1}$ Research Scholar, Department of Mechanical Engineering, Indian Institute of Technology Guwahati, Guwahati, Assam, India-781039 \\ ${ }^{2}$ Professor, Department of Mechanical Engineering, Indian Institute of Technology Guwahati, Guwahati, Assam, India-781039
}

\begin{abstract}
In the present work a simple and efficient least squares method is implemented for accurate estimation of notch stress intensity factors (NSIFs) of sharp V-notches. Finite element (FE) stress components near a notch tip is used in the present method for determining the NSIFs. Pure mode I and mixed mode (I/II) examples are considered for numerical investigations. The mixed mode stress components are disintegrated into opening mode and shear mode stress components to separate out the mode I and mode II singularities. Thereafter, least squares method is implemented to calculate mixed mode NSIFs. The present method is easy to incorporate in existing standard finite element codes. The results obtained by the present method are found to be in good agreement with the published data.
\end{abstract}

Keywords: Notch stress intensity factor, Sharp V-notch, Finite element method, Least squares method

\section{Introduction}

Many engineering components and structures contain sharp or rounded V-notches and reentrant corners. Some notches are knowingly made in engineering elements to serve some specific purposes. Moreover, notches may develop due to manufacturing defects also. Cracks are also particular case of sharp notches. When in comparison to the other dimensions of notch, the notch tip radius is very small, it can be consider as sharp Vnotch. The presence of notches may substantially increase local stresses at the notch tip. Due to severe local stresses, the components may get damaged well below its expected yield strength. The scenario becomes even worse in case of variable loads. Therefore, a reliable stress analysis is desired in the domain near to the notch tip to avoid catastrophic failures of engineering components and structures. Williams [1] showed that stress singularity exist for 2D elastic configuration containing sharp V-notches. In linear elastic fracture mechanics, notch stress intensity factors (NSIFs) can be used to describe the singular stress field at the notch tip. The singular stress field near at any point $r$ near the notch tip can be written as $K r^{1-\lambda} f_{i j}(\theta), \lambda-1$ is the order of stress singularity and $f_{i j}(\theta)$ are angular components of stress field. $K$ is defined as notch stress intensity factor of the stress field. NSIFs are used by researches as brittle fracture criteria [2-4] and fatigue strength assessment $[5,6]$. Therefore, many authors studied the V-notch problems and tried to establish different methods such as semi-analytical methods $[7,8]$, numerical methods [9-17] and experimental methods $[18,19]$ for calculating NSIFs. For complicated problems numerical methods are mostly used. The various numerical methods available are boundary collocation method (BCM) [9], boundary element method (BEM) $[10,11]$ and finite element method (FEM) [12-17]. Chan [7] determined NSIFs for single and double edge notch problems using body force method. Zhao and Hahn [8] proposed a methodology for determining NSIFs of sharp V-notches from the SIFs of a crack problem. Ayatollahi and Nejati [12] determined the NSIFs and higher order Williams coefficients using overdeterministic method. Lazzarin [13] proposed a new methodology for computation of NSIFs from averaged strain energy density. Ju and Chung [14] and Liu et al [15] used least squares method for evaluation of NSIFs of sharp V-notched bodies. In numerical simulation shown by Ju and Chung [14] showed that the error in NSIFs are minimum if the more than two number of Williams coefficients are used. Liu et al [15] proposed a new least squares method for finding NSIFs. In their approach only one Williams coefficient is used, however, the procedure for determining the mixed mode (I/II) NSIFs is very complicated. Other than conventional FE method, Treifi et al [16] implemented fractal-like finite element method and Yi [17] used extended finite element method for evaluation of NSIFs. The least square method proposed by Liu et all [15] is the simplest, robust and very efficient for problems having one stress singularity. In the present 
investigation, this method has been further explored with different components of stresses for accurate evaluation of NSIFs of sharp V-notched configurations under pure mode I conditions (only mode I stress singularity is present). However, the procedure is complicated for cases with two singularities (mixed mode cases have both modes I and II singularities). Therefore, the mixed mode stress components are disintegrated into opening mode and shear mode stress components as suggested by Ishikawa et al [20] so that the simple method proposed by Liu et al [15] could be used as well for mixed mode problems easily.

The outline of the rest of the paper is organized as follows: the formulations for the NSIFs calculation using least squares method is described in Section 2. Section 3 presents some numerical examples. Finally, the conclusions from this work are given in Section 4.

\section{Theoretical background}

\subsection{Stress field due to sharp V-notch}

Elastic stress field at any nearby point of a sharp V-notch having notch angle $\gamma$ (Fig. 1) can be expressed by Eq. (1) using Williams approach [1]

$$
\left\{\begin{array}{l}
\sigma_{x x} \\
\sigma_{y y} \\
\sigma_{x y}
\end{array}\right\}=K_{I} A^{\prime} r^{\lambda_{1}^{I}-1}\left\{\begin{array}{l}
f_{x x} \\
f_{y y} \\
f_{x y}
\end{array}\right\}+K_{I I} B^{\prime} r^{\lambda_{1}^{I I}-1}\left\{\begin{array}{l}
g_{x x} \\
g_{y y} \\
g_{x y}
\end{array}\right\}
$$

where $K_{I}$ and $K_{I I}$ denote modes I and II NSIFs respectively, $(r, \theta)$ are the polar coordinates, $A_{1}$ and $B_{1}$ are Williams coefficients corresponding to the singularity terms and $\lambda_{1}^{I}, \lambda_{1}^{I I}$ are eigenvalues for modes I and II obtained from the characteristic Eq. (2). The angular functions $f_{i j}$ and $g_{i j}(i, j=x, y)$ are given by Eqs. ( 3 and 4 ). $A^{\prime}$ and $B^{\prime}$ can be obtained using Eqs. (5 and 6).

$$
\begin{gathered}
\lambda_{1}^{I} \sin 2 \alpha+\sin 2 \lambda_{1}^{I} \alpha=0 \\
\lambda_{1}^{I I} \sin 2 \alpha-\sin 2 \lambda_{1}^{I I} \alpha=0 \\
\left\{\begin{array}{l}
f_{x x} \\
f_{y y} \\
f_{x y}
\end{array}\right\}=\left\{\begin{array}{c}
\left\{\begin{array}{c}
\left(2+\lambda_{1}^{I} \cos 2 \alpha+\cos 2 \alpha \lambda_{1}^{I}\right) \cos \left(\lambda_{1}^{I}-1\right) \theta \\
-\left(\lambda_{1}^{I}-1\right) \cos \left(\lambda_{1}^{I}-3\right) \theta
\end{array}\right\} \\
\left\{\begin{array}{c}
\left(2-\lambda_{1}^{I} \cos 2 \alpha-\cos 2 \alpha \lambda_{1}^{I}\right) \cos \left(\lambda_{1}^{I}-1\right) \theta \\
+\left(\lambda_{1}^{I}-1\right) \cos \left(\lambda_{1}^{I}-3\right) \theta
\end{array}\right\} \\
\left\{\begin{array}{c}
-\left(\lambda_{1}^{I} \cos 2 \alpha+\cos 2 \alpha \lambda_{1}^{I}\right) \sin \left(\lambda_{1}^{I}-1\right) \theta \\
+\left(\lambda_{1}^{I}-1\right) \sin \left(\lambda_{1}^{I}-3\right) \theta
\end{array}\right\}
\end{array}\right.
\end{gathered}
$$

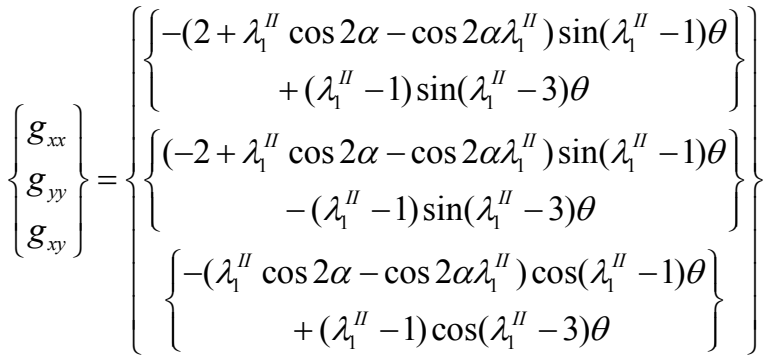

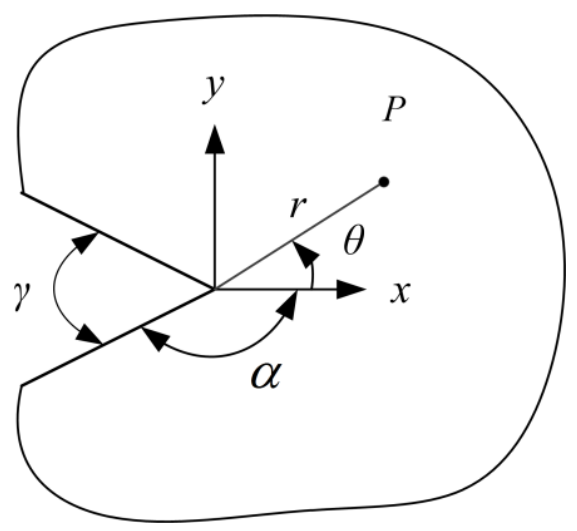

Fig. 1. Notch geometry.

$$
\begin{gathered}
A^{\prime}=\frac{1}{\sqrt{2 \pi}\left(1+\lambda_{1}^{I}-\lambda_{1}^{I} \cos 2 \alpha-\cos 2 \alpha \lambda_{1}^{I}\right)} \\
B^{\prime}=\frac{1}{\sqrt{2 \pi}\left(-1+\lambda_{1}^{I I}-\lambda_{1}^{I I} \cos 2 \alpha+\cos 2 \alpha \lambda_{1}^{I I}\right)}
\end{gathered}
$$

The variations of the dominating eigenvalues for both mode I and mode II are plotted in Fig. 2. From Fig. 3 it is clear that the geometry is symmetric about $x$ axis, therefore the stress components of the FE solution at a point $P(x, y)$ can be decomposed into mode I (opening mode) and mode II (shear mode) stress components as [20]

$$
\left\{\begin{array}{l}
\sigma_{x x}^{I} \\
\sigma_{y y}^{I} \\
\sigma_{x y}^{I}
\end{array}\right\}=\frac{1}{2}\left\{\begin{array}{l}
\sigma_{x x}^{u}+\sigma_{x x}^{l} \\
\sigma_{y y}^{u}+\sigma_{y y}^{l} \\
\sigma_{x y}^{u}-\sigma_{x y}^{l}
\end{array}\right\} \text { and }\left\{\begin{array}{l}
\sigma_{x x}^{I I} \\
\sigma_{y y}^{I I} \\
\sigma_{x y}^{I I}
\end{array}\right\}=\frac{1}{2}\left\{\begin{array}{l}
\sigma_{x x}^{u}-\sigma_{x x}^{l} \\
\sigma_{y y}^{u}-\sigma_{y y}^{l} \\
\sigma_{x y}^{u}+\sigma_{x y}^{l}
\end{array}\right\}
$$

where $\sigma_{x x}^{I}, \sigma_{y y}^{I}, \sigma_{x y}^{I}$ and $\sigma_{x x}^{I I}, \sigma_{y y}^{I I}, \sigma_{x y}^{I I}$ are finite element stress components, where suffix $I$ and $I I$ indicate opening mode and shear mode. Suffix $u$ and $l$ represents stress components at points $P(x, y)$ and $P^{\prime}(x,-y)$.

Therefore, the mode I and mode II stress components can be written as 


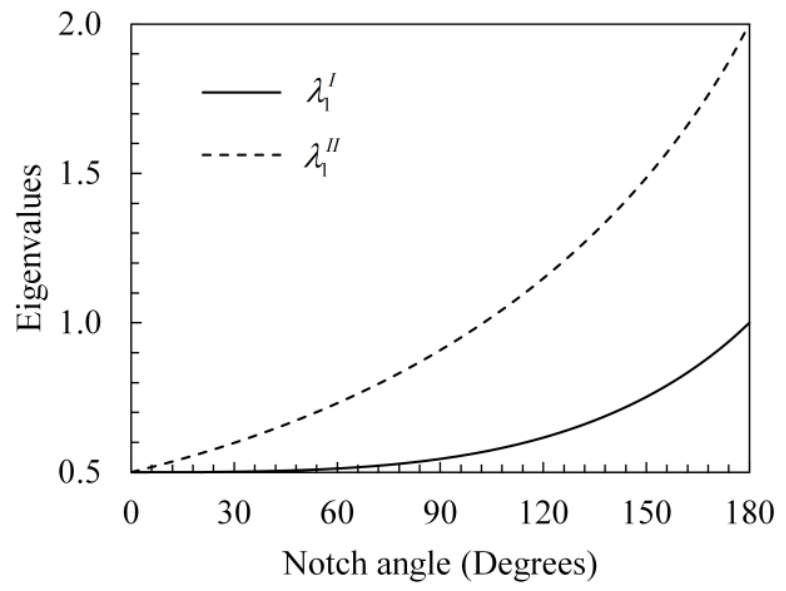

Fig. 2. Dominant eigenvalues for Modes I and II.

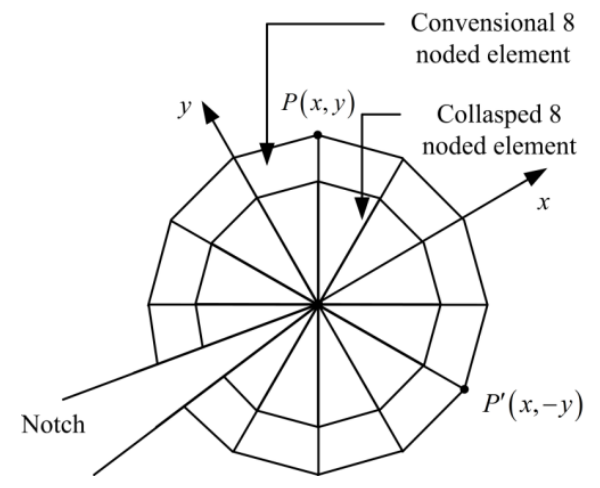

Fig. 3. A typical mesh around the notch tip.

$$
\begin{aligned}
& \left\{\begin{array}{l}
\sigma_{x x}^{I} \\
\sigma_{y y}^{I} \\
\sigma_{x y}^{I}
\end{array}\right\}=K_{I} A^{\prime} r^{\lambda_{1}^{I}-1}\left\{\begin{array}{l}
f_{x x} \\
f_{y y} \\
f_{x y}
\end{array}\right\} \\
& \left\{\begin{array}{l}
\sigma_{x x}^{I I} \\
\sigma_{y y}^{I I} \\
\sigma_{x y}^{I I}
\end{array}\right\}=K_{I I} B^{\prime} r^{\lambda_{1}^{I I}-1}\left\{\begin{array}{l}
g_{x x} \\
g_{y y} \\
g_{x y}
\end{array}\right\}
\end{aligned}
$$

For the present analysis, mode I NSIFs is determined from the stress component $\sigma_{y y}^{I}$ and mode II NSIFs from $\sigma_{x y}^{I I}$.

\subsection{Determination of NSIFs by least squares method}

In the elastic plane problem, the stress component at the vicinity of the notch tip $\sigma_{i j} \quad(i j=y y$ for symmetric deformation mode and $x y$ for anti-symmetric deformation mode) can be expressed as

$$
\sigma_{i j}=K_{k} A_{i j}{\lambda^{\lambda_{1}^{k}-1}}^{(k=I, I I)}
$$

where $A_{i j}=A^{\prime} f_{y y}$ for mode I and $A_{i j}=B^{\prime} g_{x y}$ for mode II conditions. Taking logarithm at both sides of Eq. (6)

$$
\ln \left|\sigma_{i j}\right|=\ln \left|K_{k}\right|+\ln \left|A_{i j}\right|+\left(\lambda_{1}^{K}-1\right) \ln r
$$

In order to determine the NSIFs accurately, $n$ number of nodes are considered in the direction $\theta=60^{\circ}$ for mode I and $\theta=0^{\circ}$ for mode II. The error in FE stresses and the analytical stresses can be given by

$$
R=\sum_{n=1}^{N}\left[\ln \left|\sigma_{i j n}\right|-\ln \left|K_{k}\right|-\ln \left|A_{i j}\right|-\left(\lambda_{1}^{K}-1\right) \ln r_{n}\right]^{2}
$$

Using least squares method, partial differentiations of the function $R$, with respect to $\ln \left|K_{k}\right|$ must be equal to zero, i.e.

$$
\frac{\partial R}{\partial \ln \left|K_{k}\right|}=0
$$

From Eqs. (12) and (13), one can get

$$
-2 \sum_{n=1}^{N}\left[\ln \left|\sigma_{i j n}\right|-\ln \left|K_{k}\right|-\ln \left|A_{i j}\right|-\left(\lambda_{1}^{K}-1\right) \ln r_{n}\right]=0
$$

From Eq. (14), finally the NSIFs can be obtained as

$$
K_{k}=\frac{\exp \left[\frac{1}{N} \sum_{n=1}^{N} \ln \left|\sigma_{i j n}\right| r_{n}^{1-\lambda_{1}^{k}}\right]}{\left|A_{i j}\right|}
$$

The normalized notch stress intensity factors can be described as

$$
F_{I}=\frac{K_{I}}{\sigma \sqrt{\pi} a^{\left(1-\lambda_{1}^{I}\right)}} \text { and } F_{I I}=\frac{K_{I I}}{\sigma \sqrt{\pi} a^{\left(1-\lambda_{1}^{I I}\right)}}
$$

\section{Results and discussions}

In this section, numerical examination for determination of mode I and mixed mode (I/II) NSIFs for bench mark problems is carried out. Eight node quadrilateral elements are modeled in ANSYS for finite element analysis. Three problems viz. (a) Double Edge Notched plates under uniaxial Tension (DENT), (b) Center Notched plates under uniaxial Tension (CNT) and (c) Angled Double Edge Notched plates under uniaxial Tension (ADENT) are considered for present analysis. First two Problems are examples of pure mode I condition and third problem is example of mixed mode condition. Plane stress conditions are assumed for all the problems.

Percentage relative error is calculated as

$$
\% \text { rel. error }=\left|\frac{\text { comp. value-ref. value }}{\text { ref. value }}\right| \times 100
$$

where the 'comp. value' refers to NSIFs obtained using present method and 'ref. value' is the available analytical or numerical value. 


\subsection{Mode I examples: Double Edge and Center Notched plates under uniaxial Tension}

The geometry and loading considered for both DENT and CNT are as follows: $w=10, h=10$ and $\sigma=1$ as shown in Fig. 4. The notch length $a=4$, notch angles $\gamma=0^{\circ}, 30^{\circ}, 60^{\circ}$ and $90^{\circ}$ are considered. Material properties considered are Young's modulus $E=1$ and Poisson's ratio $v=0.25$. The dimensions and loading are considered to be self-consistent. Due to symmetry in geometry as well as in loading conditions one quarter of the geometry is modelled for both DENT and CNT specimens with appropriate boundary conditions show in Fig. 4. Fig. 5 shows a typical FE mesh used for mode I problems containing 529 elements (NE) and 1700 nodes (NN). 10 number of the consecutive corner nodes along the line $\theta=60^{\circ}$ are considered and NSIFs are calculated using the stress component $\sigma_{y y}$. The first four nodes are not selected because the near tip elements may have large errors. Mode I NSIFs can be calculated using Eq. (15).

For convergence study, DENT and CNT geometries with notch length $a=4$ and notch angle $\gamma=60^{\circ}$ are considered. Three meshes are created as shown in Fig. 6 with increasing number of elements and the results are listed in Tables 1 and 2. It is found that with each mesh refinement the error in NSIFs are decreasing. Therefore, for all the problems the fine mesh (notch tip element size $=0.0033$ ) is employed. The NSIFs for DENT and CNT for various notch angles are listed in Tables 3 and 4 . The NSIFs obtained are found to be very accurate.
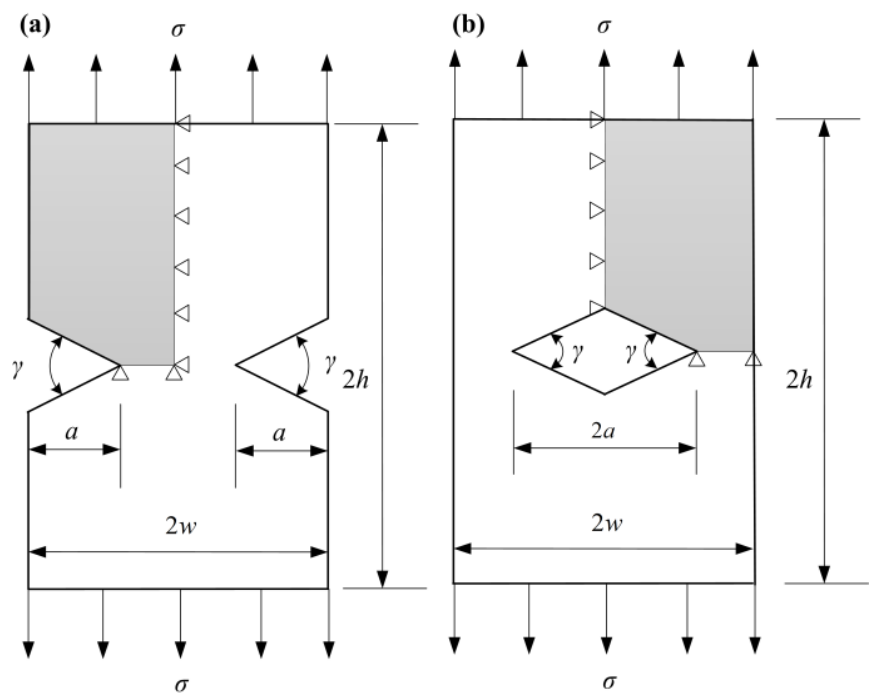

Fig. 4. (a) Double Edge Notched plate under uniaxial Tension (DENT) and (b) Center Notched plate under uniaxial Tension (CNT) specimens. (a)

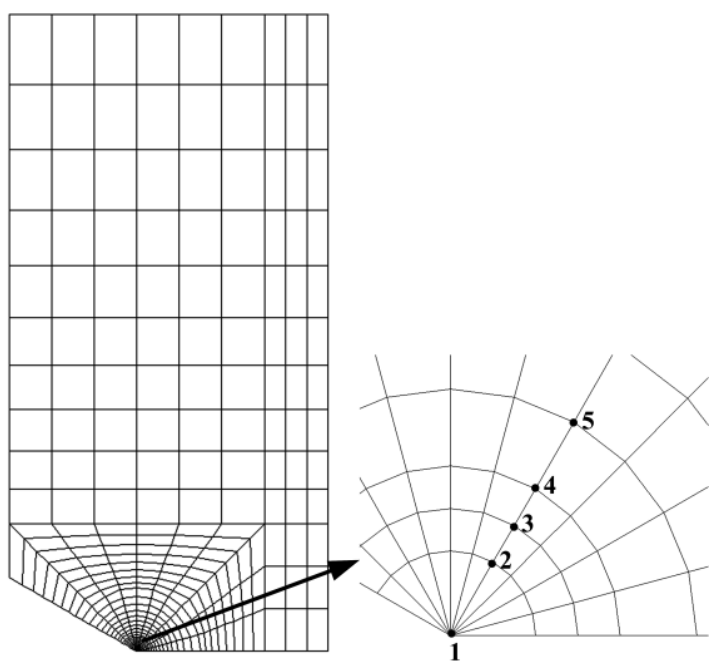

Fig. 5. (a) Typical FE mesh used for mode I specimens $\left(\gamma=60^{\circ}\right)$ and (b) enlarge view of the notch tip portion.

Table 1. Convergence for DENT specimen $(a / w=0.4$,

$$
\left.\gamma=60^{\circ}\right) \text {. }
$$

\begin{tabular}{ccclll}
\hline $\begin{array}{c}\text { Notch tip } \\
\text { element } \\
\text { size }\end{array}$ & NE & NN & & $F_{I}$ & \\
\cline { 4 - 6 } & & & Present & Ref [12] & \%rel. err \\
\hline 0.0400 & 286 & 957 & 1.2159 & & 1.49 \\
0.0133 & 340 & 1141 & 1.2036 & 1.198 & 0.47 \\
0.0033 & 529 & 1700 & 1.2004 & & 0.20 \\
\hline
\end{tabular}

Table 2. Convergence for CNT specimen ( $a / w=0.4$,

$$
\left.\gamma=60^{\circ}\right) \text {. }
$$

\begin{tabular}{|c|c|c|c|c|c|}
\hline \multirow{2}{*}{$\begin{array}{l}\text { Notch tip } \\
\text { element } \\
\text { size }\end{array}$} & \multirow{2}{*}{$\mathrm{NE}$} & \multirow{2}{*}{$\mathrm{NN}$} & \multicolumn{3}{|l|}{$F_{I}$} \\
\hline & & & Present & $\operatorname{Ref}[16]$ & $\%$ rel. err \\
\hline 0.0400 & 286 & 957 & 1.2852 & & 1.92 \\
\hline 0.0133 & 340 & 1141 & 1.2722 & 1.261 & 0.89 \\
\hline 0.0033 & 529 & 1700 & 1.2709 & & 0.79 \\
\hline
\end{tabular}

Table 3. NSIFs for DENT specimens for different $\gamma$.

\begin{tabular}{clll}
\hline \multirow{2}{*}{$\gamma\left({ }^{\circ}\right)$} & $F_{I}$ & & \\
\cline { 2 - 4 } & Present & Ref [16] & Ref. [12] \\
\hline 0 & 1.1368 & 1.132 & 1.133 \\
30 & 1.1481 & 1.143 & 1.144 \\
60 & 1.2004 & 1.191 & 1.198 \\
90 & 1.3234 & 1.323 & 1.322 \\
\hline
\end{tabular}

Table 4. NSIFs for CNT specimens for different $\gamma$.

\begin{tabular}{cll}
\hline \multirow{2}{*}{$\gamma\left({ }^{\circ}\right)$} & $F_{I}$ & \\
\cline { 2 - 3 } & Present & Ref. [16] \\
\hline 0 & 1.1150 & 1.109 \\
30 & 1.1575 & 1.151 \\
60 & 1.2709 & 1.261 \\
90 & 1.4939 & 1.499 \\
\hline
\end{tabular}


(a)

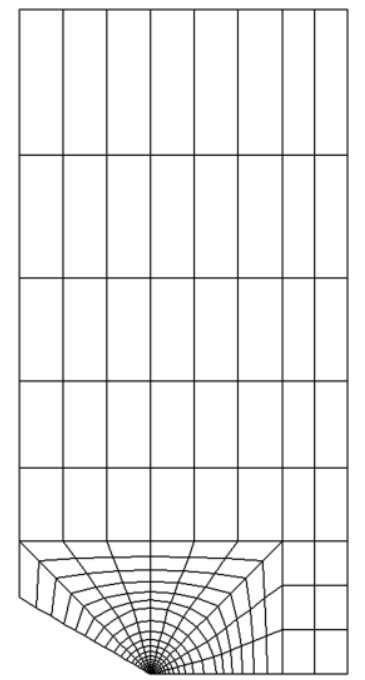

$\mathrm{NE}=286 ; \mathrm{NN}=957$ (b)

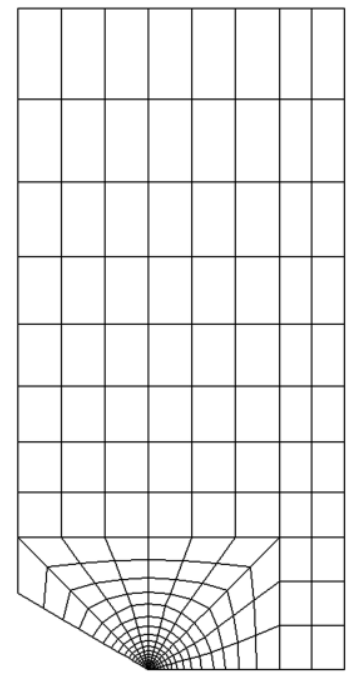

$\mathrm{NE}=340 ; \mathrm{NN}=1141$ (c)

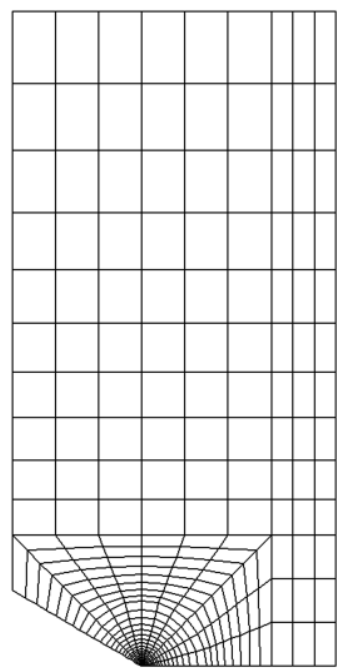

$\mathrm{NE}=529 ; \mathrm{NN}=1700$

Fig. 6. Sequence of FE meshes used for DENT and CNT specimens for convergence study $\left(\gamma=60^{\circ}\right)$.

\subsection{Mixed Mode (I/I) examples: Double Angled Edge Notched plates under uniaxial Tension}

The geometry and loading for Double Angled Edge Notched plates under uniaxial Tension (ADENT) is as follows: $w=10, h=25$ and $\sigma=1$ as shown in Fig. 7 . The notch length $a=4$, notch angles $\gamma=0^{\circ}, 30^{\circ}, 60^{\circ}$ and $90^{\circ}$ and notch inclination angle $\beta=0^{\circ}, 15^{\circ}, 30^{\circ}$ and $45^{\circ}$ are considered for the present analysis. For $\beta=0^{\circ}$ the specimen will be under pure mode I condition while for other angles of $\beta$, the specimen will be under mixed mode conditions. Young's modulus and Poisson's ratio are considered as $E=1$ and $v=0.25$. The dimensions and loading are considered to be self-consistent. Because of the symmetry in geometry and loading conditions one half of the geometry is modelled for FE analysis. For calculating $F_{I}, \sigma_{y y}$ at 10 consecutive nodes along each

line $\theta=60^{\circ} /-60^{\circ}$ are considered. In the similar way, $\sigma_{x y}$ at 10 number of nodes along $\theta=0^{\circ}$ are considered for finding $F_{I I}$. In mixed mode the starting node number is taken as 4. A typical FE mesh for $\beta=15^{\circ}$ and $\gamma=30^{\circ}$ is shown in Fig. 7 containing 957 elements (NE) and 3113 nodes (NN). The normalized NSIFs $F_{I}$ and $F_{I I}$ for various ADENT specimens are listed in Tables 5-7. The effect of notch angle, notch inclination angle and notch length can be observed in Tables 5-7. The results obtained in the present method are compared with available results and good agreement with the literature is found.

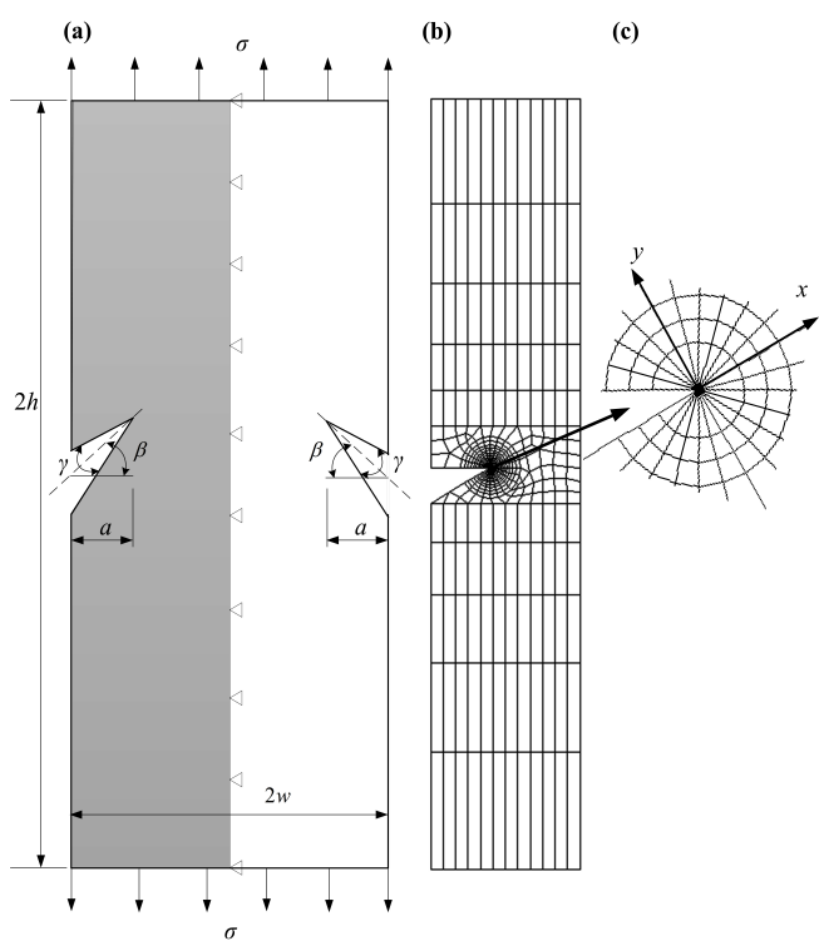

Fig. 7. (a) Angled Single Edge Notched plate under uniaxial Tension (ADENT), (b) typical FE mesh used for mixed mode specimens $\left(\beta=15^{\circ}\right.$ and $\left.\gamma=30^{\circ}\right)$ and (c) enlarge view of the notch tip portion.

Table 5. Values of $F_{I}$ and $F_{I I}$ for $a / w=0.4$ and $\beta=15^{\circ}$.

\begin{tabular}{llll}
\hline$\gamma\left({ }^{\circ}\right)$ & & $F_{I}$ & $F_{I I}$ \\
\hline \multirow{3}{*}{0} & Present & 1.0980 & 0.1806 \\
& Ref [7] & - & - \\
& Ref [12] & 1.0979 & 0.1789 \\
\hline \multirow{3}{*}{30} & Present & 1.1077 & 0.2486 \\
& Ref [7] & 1.107 & 0.248 \\
& Ref [12] & 1.1076 & 0.2474 \\
\hline \multirow{3}{*}{60} & Present & 1.1526 & 0.3387 \\
& Ref [7] & 1.153 & 0.338 \\
& Ref [12] & 1.1531 & 0.3373 \\
\hline \multirow{3}{*}{90} & Present & 1.2583 & 0.4269 \\
& Ref [7] & 1.259 & 0.426 \\
& Ref [12] & 1.2591 & 0.4241 \\
\hline
\end{tabular}


Table 6. Values of $F_{I}$ and $F_{I I}$ for $a / w=0.4$ and $\gamma=30^{\circ}$.

\begin{tabular}{clll}
\hline$\beta\left(^{\circ}\right)$ & & $F_{I}$ & $F_{I I}$ \\
\hline \multirow{3}{*}{0} & Present & 1.1481 & 0.000 \\
& Ref [7] & - & - \\
& Ref [12] & 1.144 & 0.000 \\
\hline \multirow{3}{*}{15} & Present & 1.1077 & 0.2486 \\
& Ref [7] & 1.107 & 0.248 \\
& Ref [12] & 1.1076 & 0.2474 \\
\hline \multirow{3}{*}{30} & Present & 1.0000 & 0.4522 \\
& Ref [7] & 1.000 & 0.4522 \\
& Ref [12] & 1.0005 & 0.4491 \\
\hline \multirow{3}{*}{45} & Present & 0.8380 & 0.5740 \\
& Ref [7] & 0.839 & 0.574 \\
& Ref [12] & - & - \\
\hline
\end{tabular}

Table 7. Values of $F_{I}$ and $F_{I I}$ for $\beta=30^{\circ}$ and $\gamma=60^{\circ}$.

\begin{tabular}{llll}
\hline$a / w$ & & $F_{I}$ & $F_{I I}$ \\
\hline \multirow{4}{*}{0.2} & Present & 0.9994 & 0.5912 \\
& Ref [7] & 1.000 & 0.589 \\
& Ref [12] & - & - \\
\hline \multirow{2}{*}{0.4} & Present & 1.0238 & 0.5968 \\
& Ref [7] & 1.024 & 0.596 \\
& Ref [12] & 1.0263 & 0.5944 \\
\hline \multirow{2}{*}{0.6} & Present & 1.1396 & 0.6307 \\
& Ref [7] & 1.139 & 0.631 \\
& Ref [12] & - & - \\
\hline
\end{tabular}

\section{Conclusions}

In this paper, a least squares method proposed by Liu et al. [15] is employed for determination of the mode I with different stress components. The mixed mode stress components are disintegrated into opening mode and shear mode stress components to separate out the mode I and mode II singularities. As a result, the stress terms will have only one singularity (either mode I or mode II) even in case of mixed mode problems. Therefore, the mixed problem can be considered as single stress singularity and can be proceed accordingly. Two mode I examples and one mixed mode (I/II) example are considered for explaining the working of the present method. The results obtained are in good agreement with the reference values. The method is simple, efficient and easy to implement in the existing finite element codes for simultaneous estimation of mode I and mixed mode (I/II) NSIFs. The present method can be extended for blunt $\mathrm{V}$-notch problems also.

\section{References}

1. M.L. Williams, J. Appl. Mech. 19, 526-528 (1952).

2. M.L. Dunn, W. Suwito, Int J Solids Struct 45, 646834, 29, 3873-3883 (1997).

3. A. Seweryn, Eng. Fract. Mech. 47, 673-81 (1994).

4. Carpinteri A. Eng. Fract. Mech. 26, 143-55 (1987).

5. P. Lazzarin, P. Livieri, Int. J. Fatigue 23 225-32 (2001).

6. C. Fischer,W. Fricke,C.M. Rizzo, Int. J. Fatigue 84, 59-66 (2016).

7. D.H. Chen, Int. J. Fract. 70, 81-97 (1995).

8. Z. Zhao, H.G. Hahn, 43, 511-8 (1992).

9. Gross B, Mendelson A, Int. J. Fract. Mech. 8, 26776 (1972).

10. C Cheng, Z Niu, H Zhou, N Recho, Eng. Anal. Bound. Elem. 33, 1145-51 (2009).

11. Z Niu, C Cheng, J Ye, N Recho, Int. J. Solids Struct. 46, 2999-3008 (2009).

12. M.R. Ayatollahi, M. Nejati, Int. J. Mech. Sci. 53, 164-77 (2011).

13. P. Lazzarin, F. Berto, M Zappalorto, Int. J. Fatigue 32, 1559-67 (2010).

14. S.H. Ju, H.Y. Chung, Int. J. Fract. 148, 169-83 (2007).

15. Y. Liu, Z. Wu, Y. Liang, X. Liu, Eng. Fract. Mech. 75, 4793-803 (2008).

16. M. Treifi, S.O. Oyadiji, D.K.L. Tsang, Eng. Fract. Mech. 76, 2091-2108 (2009).

17. G. Yi, T. Yu, T. Quoc, C. Ma, S. Hirose, Theor. Appl. Fract. Mech. 89, 35-44 (2017).

18. M.R. Ayatollahi, M. Nejati, Mater Des 32, 561-9 (2011).

19. T. Kondo, Y. Kurabe, T. Sasaki, T. Kurahashi, Y. Miyashita, Eng. Fract. Mech. 124-125, 248 -61 (2014).

20. H. Ishikawa, H. Kitagawa, H. Okamura, Mechanical Behaviour of Materials, 3, 447-55 ICM (1979). 